Supporting Information for

\title{
Epoxidized block and statistical copolymers reinforced by organophosphorous-titanium-silicon hybrid nanoparticles: morphology, thermal and mechanical properties
}

\author{
Faezeh Hajiali, Saeid Tajbakhsh, Milan Marić* \\ Department of Chemical Engineering, McGill University, 3610 University St, Montreal, H3A 0C5, Quebec, Canada \\ Correspondence to: \\ M. Marić (E-mail: milan.maric@mcgill.ca)
}

\section{Contents}

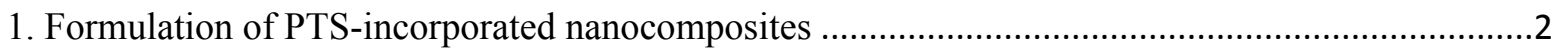

2. Multiple Gaussian fitting for Estimation of Dead Polymer Chains …..............................................

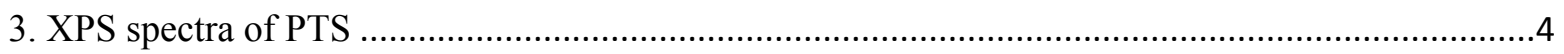

4. Images of Cured Poly(GMA-stat-C13MA) and Poly(GMA-b-C13MA) ........................................ 
1. Formulation of PTS-incorporated nanocomposites

Table S1. Formulation of poly(GMA-stat-C13MA)/PTS and poly(GMA-b-C13MA)/PTS nanocomposites

\begin{tabular}{ccc}
\hline Sample ID & Polymer (wt\%) & PTS (wt\%) \\
\hline S-0 & poly(GMA-stat-C13MA) (100) & 0 \\
\hline S-0.5 & poly(GMA-stat-C13MA) (99.5) & 0.5 \\
\hline S-2 & poly(GMA-stat-C13MA) (98) & 2 \\
\hline S-4 & poly(GMA-stat-C13MA) (96) & 4 \\
\hline B-0 & poly(GMA-b-C13MA) (100) & 0 \\
\hline B-4 & poly(GMA- $b-C 13 M A)(96)$ & 4 \\
\hline
\end{tabular}

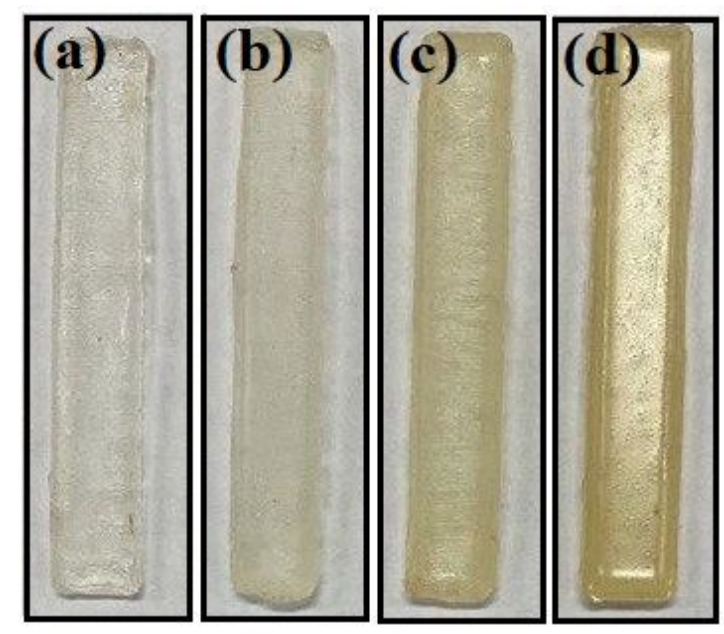

Figure S1. Picture of poly(GMA-stat-C13MA) incorporated with PTS: (a) S-0, (b) S-0.5, (c) S-2, (d) S-4 nanocomposites. 


\section{Multiple Gaussian fitting for Estimation of Dead Polymer Chains}

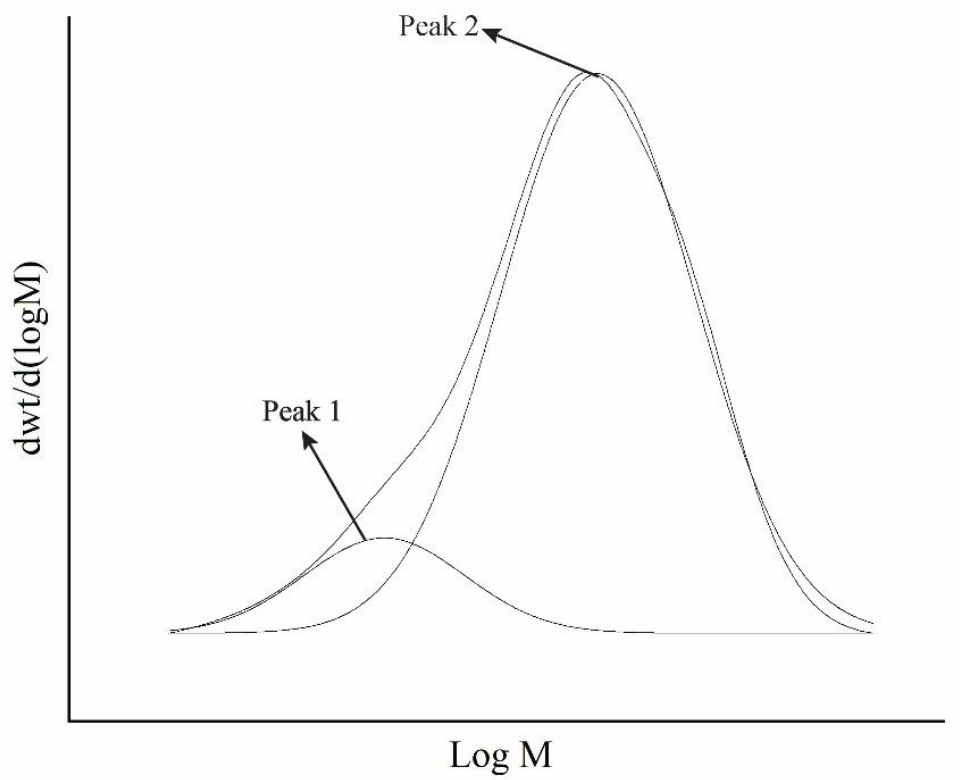

Figure S2. Multiple Gaussian peak fitting for the estimation of the percentage of dead chains for poly(GMA) macroinitiator after precipitation in methanol. Peak1 and peak 2 represent the Isolated Gaussian peaks for the dead chains and active polymer chains, respectively. 


\section{XPS spectra of PTS}

(a)

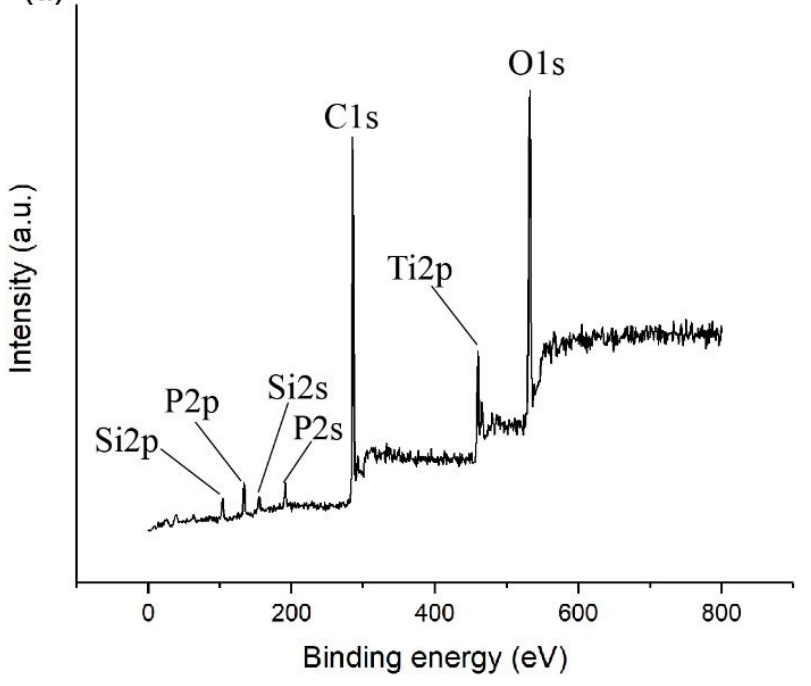

(b)

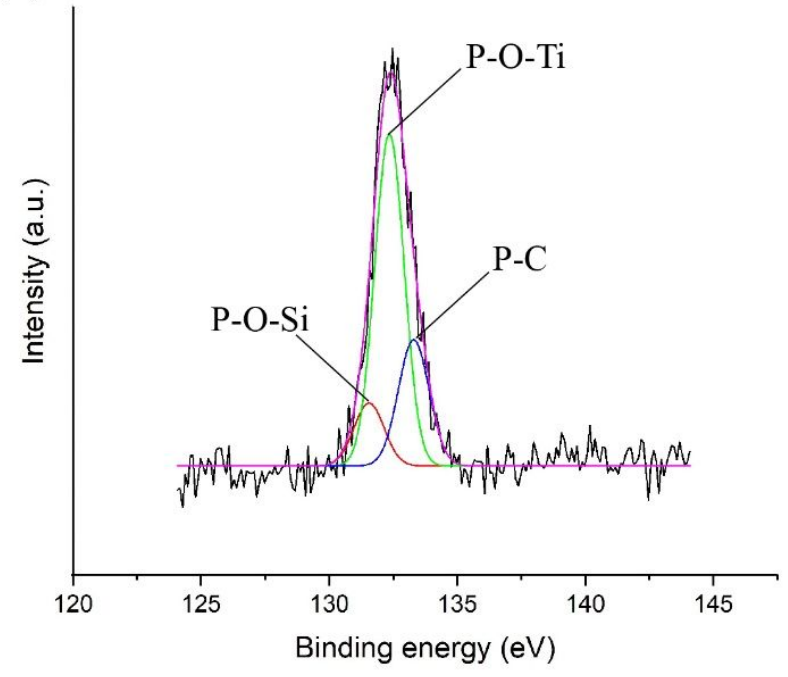

Figure S3. (a) XPS spectra and (b) P 2p spectra of PTS. 
4. Images of Cured Poly(GMA-stat-C13MA) and Poly(GMA-b-C13MA)

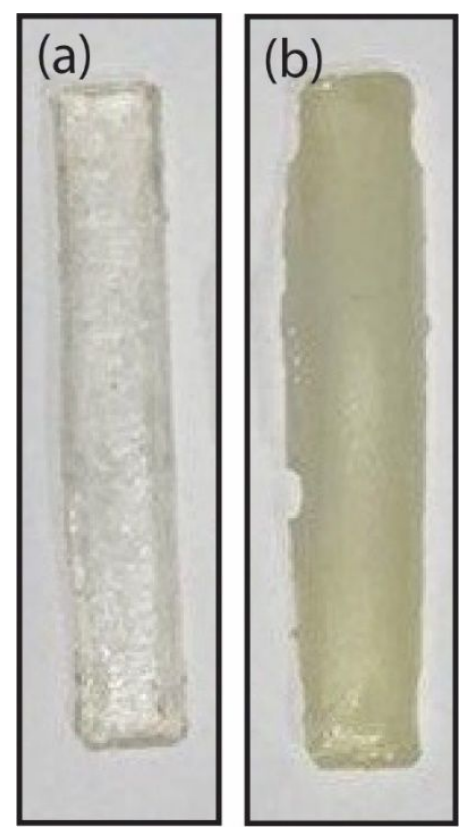

Figure S4. Images of the samples demonstrating (a) the transparency of cured poly(GMA-stat-C13MA) versus (b) opaque cured poly(GMA- $b$-C13MA). 